\title{
Great challenges in molecular medicine: toward personalized medicine
}

\author{
Masaru Katoh * \\ Katoh's Unit, National Cancer Center, Tokyo, Japan \\ *Correspondence: mkatoh-kkr@umin.ac.jp \\ Edited by: \\ Craig M. Walsh, University of California, Irvine, USA
}

Keywords: bio-bank, database, knowledgebase, clinical sequencing, big data, WNT, FGF, Hedgehog

\section{GENE HUNTING, SIGNALING NETWORK, AND MOLECULAR MEDICINE}

In the 20th century, most researchers investigated WNT signaling in cell and developmental biology by using model animals, such as Drosophila, Xenopus, and mouse. However, I was confident that WNT signaling should be investigated for clinical application by using human samples or cell lines. In 1998, I, together with post-doctoral fellows, started a human WNTome project to comprehensively clone and characterize novel human genes encoding WNT signaling molecules and to establish a "human WNT research" platform (Figure 1A). My group reported the molecular cloning and characterization of FZD1, FZD3, FZD4, FZD6, FZD7, FZD8, FZD10, GIPC2, GIPC3, MFRP, NKD1, NKD2, VANGL1, WNT3A, WNT5B, WNT6, WNT7B, WNT8A, WNT9A (WNT14), WNT9B (WNT14B), and WNT10A as the major products of the human WNTome project [reviewed in Katoh (2002a)] and of other novel human genes, such as FGF20, RhoU, $R h o V$, and SOX17, as byproducts of the human WNTome project.

Most human genes that encode WNT signaling components had been cloned and characterized by 2002, whereas thousands of novel human genes outside of the WNT field still remained to be discovered. In 2003, colleagues and I started a post-WNTome project to identify and characterize novel human genes encoding adhesion molecules, transmembrane proteins, epigenetic regulators, and transcription factors (Figure 1A). My group reported in silico identification and characterization of novel human genes, such as ANO1 (TMEM16A), ANO2 (TMEM16B), ANO3 (TMEM16C),
ANO4 (TMEM16D), ANO5 (TMEM16E), ANO6 (TMEM16F), ANO7 (TMEM16G), ANO8 (TMEM16H), ASXL2, ASXL3, BCL9L, CDC50A (TMEM30A), CDC50B (TMEM30B), CDC50C (TMEM30C), CRB2, DACT1 (DAPPER1), DACT2 (DAPPER2), DIXDC1, FAT4, FMNL1, FMNL2, FMNL3, FOXR1 (FOXN5), FOXR2 (FOXN6), HES2, HES3, HES5, JMJD1C (TRIP8), JMJD2A (KDM4A), JMJD2B (KDM4B), JMJD2C (KDM4C), JMJD2D (KDM4D), KIF27, MPP7, PRICKLE1, and PRICKLE2.

The human WNTome and postWNTome projects were gene-hunting adventures that utilized molecular biology and computational biology, respectively. Inter- and intra-cellular signaling networks were simplified to a secondary picture consisting of nodes and edges. Nodes correspond to genes, mRNAs, proteins, or micro-RNAs (miRNAs), while edges correspond to their interactions. I then shifted my interest from the nodes to the edges and the whole picture. In 2007, my laboratory started a stem-cell signaling network project to elucidate mutual interactions of the WNT, FGF, Notch, Hedgehog, TGF- $\beta$, and BMP signaling cascades (Figure 1A) (Katoh and Katoh, 2007, 2009; Katoh and Nakagama, 2013).

Recently, I was appointed as the chief editor of Frontiers in Molecular Medicine, a sub- or specialty journal of Frontiers in Cell and Developmental Biology. I would like to contribute to the global scientific community through Frontiers in Molecular Medicine, which aims to address the gap between cell and developmental biology and clinical medicine and to promote development of novel diagnostics and therapeutics for a variety of human diseases, including cancers, cardiovascular diseases, diabetes mellitus, eye diseases, inflammatory bowel diseases, kidney diseases, liver diseases, neurological diseases, and respiratory diseases.

\section{MOLECULAR MEDICINE TARGETED TO THE REGULATORY SIGNALING NETWORK}

Mouse mammary tumor virus (MMTV) integrates at the Wnt1, Wnt3, Wnt10b, $\mathrm{Fgf3}, \mathrm{Fgf4}$, or Fgf10 loci and induces mammary tumorigenesis [Dickson et al., 1984; Nusse et al., 1984; reviewed in Katoh (2002b)]. WNT signals are transduced through Frizzled (FZD) receptors and LRP5/6 or ROR $1 / 2$ co-receptors at both the canonical and non-canonical signaling cascades (Katoh and Katoh, 2007). Canonical WNT signals regulate cell fate (Clevers, 2006) in co-operation with FGF and Notch signals, while non-canonical WNT signals regulate cell morphogenesis and motility (Chien et al., 2009) in co-operation with FGF, TGF- $\beta$, and Hedgehog signals. The WNT, FGF, Notch, Hedgehog, and TGF- $\beta$ signaling cascades constitute the stem cell signaling network, which orchestrates fetal development and post-natal homeostasis, whereas dysregulation of the stem cell signaling network causes a variety of hereditary and sporadic diseases (Katoh and Katoh, 2007).

Receptor tyrosine kinases (RTKs) are single-span transmembrane receptors with extracellular ligand-binding domain and intracelullar tyrosine kinase domain and are involved in growth factor signaling to downstream cascades, including the RAS-MAPK, PI3K-AKT, SRC, and PKC signaling cascades. FGFR1, FGFR2, FGFR3, and FGFR4 are receptors of the FGF family of ligands (Eswarakumar et al., 2005; Beenken and Mohammadi, 2009), and VEGFR1, VEGFR2, and VEGFR3 are receptors of the VEGF family of 


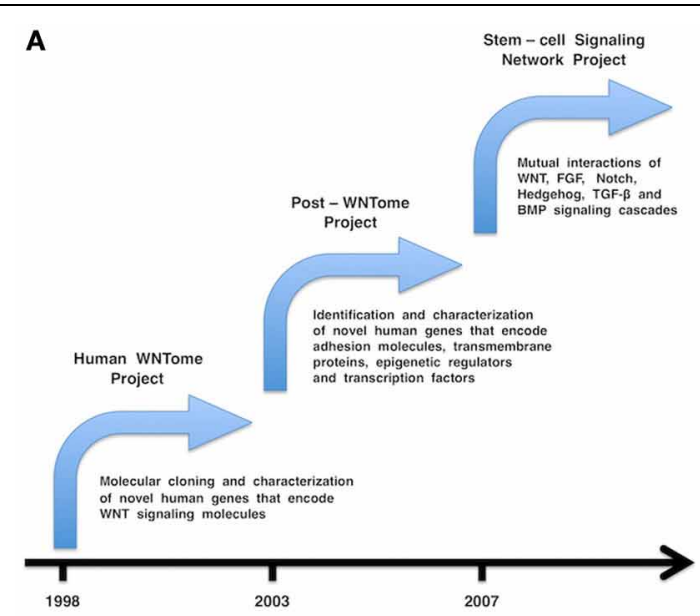

FIGURE 1 | (A) Chronology of the human WNTome, post-WNTome, and stem-cell signaling network projects. (B) Fields in molecular medicine. Regulatory signaling network, cell biology, and omics medicine are the major fields in molecular medicine. Regulatory signaling network consists of receptor tyrosine kinase (RTK), G protein-coupled receptor (GPCR), and other signaling cascades, such as FGF, VEGF, WNT, Hedgehog, Notch, TGF- $\beta$, and BMP signaling cascades. Cell biology

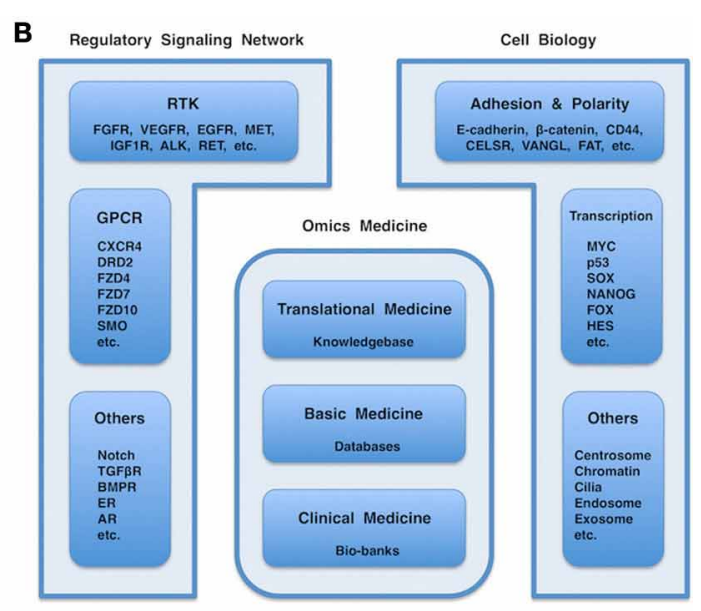

includes various important topics: cellular adhesion and polarity; centrosome biology; chromatin dynamics; endosome and exosome; and transcriptional regulation. Omics medicine consists of three layers: clinical medicine, followed by basic medicine and translational medicine. Bio-banks, databases, and a comprehensive knowledgebase are generated by clinical medicine, basic medicine, and translational medicine, respectively. ligands (Tammela et al., 2005; Ellis and Hicklin, 2008). Growing evidence indicates that the WNT, Notch, Hedgehog, and TGF- $\beta$ signaling cascades crosstalk with the FGFR signaling cascade, as well as other RTK signaling cascades. Because genetic alterations in RTKs, such as FGFRs, EGFR, HER2, MET, ALK, and RET, drive human cancers, small-molecule inhibitors and human/humanized monoclonal antibodies targeted against RTKs have been developed as cancer therapeutics (Ciardiello and Tortora, 2008; Kwak et al., 2010; Mologni, 2011; Buettner et al., 2013; Katoh and Nakagama, 2013; Li et al., 2013). VEGF antibodies are used as therapeutic agents for cancers and neovascular age-related macular degeneration (AMD) (Penn et al., 2008; Katoh, 2013b). Because clinical sequencing may reveal genetic changes underlining diseases without established therapeutics, the application of RTK inhibitors for previously unknown disease entities based on genetic alterations should be published as regular reports or case reports. Therapeutics targeting RTKs in cancers and non-cancerous diseases are important issues that will be published in Frontiers in Molecular Medicine.

$\mathrm{G}$ protein-coupled receptors (GPCRs) are seven transmembrane receptors that are linked to small G-protein signaling and other atypical signaling cascades. WNT receptors, including Frizzled-1 (FZD1), FZD2, FZD3, FZD4, FZD5, FZD6, FZD7, FZD8, FZD9, and FZD10, as well as Hedgehog signal transducer Smoothened, belong to the GPCR superfamily (Sagara et al., 1998; Koike et al., 1999; Katoh and Katoh, 2007; Lappano and Maggiolini, 2011). GPCRs are classified as class A (Rhodopsin family), class B (Secretin and Adhesion family), class C (Glutamate family), or class D (others, such as FZD/Smoothened and Taste2 family) (Lagerström and Schiöth, 2008). High-resolution structures of class A GPCRs have been reported, and those of class B GPCRs have also been recently reported (Sexton and Wootten, 2013). Small-molecule inhibitors targeted to Smoothened and monoclonal antibody targeted to FZD7 have been developed as cancer therapeutics (Katoh and Katoh, 2009; Philips et al., 2011; Arzumanyan et al., 2012; Gurney et al., 2012). Therapeutics targeting GPCRs in cancers and non-cancerous diseases are also important topics that will be published in Frontiers in Molecular Medicine.

The WNT, FGF, Notch, Hedgehog, and TGF- $\beta$ signaling network is the tip of the iceberg for the regulatory signaling network that consists of signaling cascades via RTKs, GPCRs and other receptors (Figure 1B). Mutual interactions of this regulatory signaling network should be comprehensively investigated for the development and optimization of therapeutics.

\section{MOLECULAR MEDICINE TARGETED TO CELL BIOLOGY}

Cellular adhesion, cellular polarity, cellular proliferation, cellular survival, chromatin modification, cilia formation, DNA repair, endocytosis, exocytosis, and transcriptional regulation are all major topics in cell and developmental biology and molecular medicine (Figure 1B).

Forkhead-box (FOX) family members are DNA-binding proteins with a FOX domain that consists of two wing-like loops and three $\alpha$-helices (Carlsson and Mahlapuu, 2002; Katoh and Katoh, 2004a; Hannenhalli and Kaestner, 2009). Because FOX proteins are involved in transcriptional regulation and DNA repair (Katoh et al., 2013), germ-line mutations in the FOX family of genes cause hereditary diseases, such as Axenfeld-Rieger syndrome; lymphedema-distichiasis syndrome; blepharophimosis, ptosis and epicanthus inversus syndrome; and speech and language disorder (Lehmann et al., 2003). Somatic mutations in the FOX family of genes, including gene amplifications, 
point mutations, and translocations, occur in a variety of human cancers (Katoh et al., 2013). My group identified and characterized the FOXR1 (FOXN5) gene within a cancer-associated deleted region in human chromosome 11q23.3 in 2004 (Katoh and Katoh, 2004c); Santo et al. later reported a FOXR1-MLL1 fusion caused by an intrachromosomal deletion in neuroblastoma in 2012 (Santo et al., 2012).

The Drosophila Asx gene encodes an epigenetic regulator that is associated with the Polycomb-group $(\mathrm{PcG})$ repressor complex and the trithorax-group (trxG) activator complex (Jürgens, 1985; Sinclair et al., 1998; Brock and Fisher, 2005). ASXL1 (Fisher et al., 2003), ASXL2 (Katoh and Katoh, 2003), and ASXL3 (Katoh and Katoh, 2004b), which contain ASXN, ASXH, ASXM1, ASXM2, and PHD domains, are human homologs of the Drosophila Asx. BAP1, KDM1A (LSD1), NCOA1, the retinoic acid receptors (RAR $\alpha$ and $\mathrm{RAR} \beta$ ), estrogen receptor (ER), and glucocorticoid receptor (GR) are binding partners of ASXL1. Germ-line mutations in ASXL1 occur in Bohring-Opitz syndrome, while somatic mutations in ASXL1 occur in colorectal cancer with microsatellite instability, hematological malignancies and castration-resistant prostate cancer (Gelsi-Boyer et al., 2012; Katoh, 2013a and references therein).

Extracellular DNA and circulating miRNAs are key topics in translational medicine (Wittmann and Jäck, 2010; Turchinovich et al., 2012), and epigenetics play a key role in cancerous and noncancerous diseases (Ordovás and Smith, 2010; Baylin and Jones, 2011). I recently underlined diagnostic techniques utilizing circulating miRNAs in exosomes and microsomes, therapeutics utilizing siRNAs in polymer-based hydrogel nanoparticles and therapeutics targeted to a field of epigenetic alterations (Katoh, 2013b). Manuscripts on a various aspects of cell and developmental biology that are applicable for molecular medicine are also welcome for publication in Frontiers in Molecular Medicine.

\section{THE THREE-LAYER STRUCTURE OF OMICS MEDICINE: BIO-BANKS, DATABASES, AND A COMPREHENSIVE KNOWLEDGEBASE}

Genomics, transcriptomics, proteomics and metabolomics are representative "omics" disciplines of life science that deal with the entirety of genes, transcripts, proteins, and metabolites, respectively. Omics medicine is an emerging discipline of medical science that produces large amounts of omics data on genetics, genomics, epigenetics, transcriptomics, proteomics, and metabolomics. Here, I propose my personal view on the three-layer structure of omics medicine (Figure 1B). The first layer of omics medicine corresponds to clinical medicine that involves with patients' care and clinical sampling of blood and tissues (bio-banks). The second layer of omics medicine corresponds to basic medicine that produces cutting-edge data by using conventional molecular biology technologies, as well as high-throughput omics data using microarrays and nextgeneration sequencing technologies. The third layer of omics medicine corresponds to translational medicine, which develops novel diagnostics and therapeutics. Bioinformatics used to generate curated databases from high-throughput raw data by using algorithms (techint) is classified into the second layer, while bioinformatics used to generate a knowledgebase from manuscripts and curated databases using either human intelligence or a Watsontype supercomputer (humint) is classified into the third layer. I was engaged in clinical medicine as a physician from 1986 to 1990 and in basic medicine on WNT and FGF signaling cascades from 1990 to 2002 and have been engaged in translational medicine on the WNT, FGF, Hedgehog, Notch, TGF- $\beta$ and BMP signaling cascades since 2003. The emergence of molecular biology evoked a great rotation from clinical medicine to basic medicine in the 20th century, while computer and internet technologies, an aging demography and governmental financial burdens have been promoting a rotation from basic medicine to translational medicine in the 21 st century.

Industry also consists of three layers. The first layer of industry includes agriculture, forestry, fishery, and mining; the second layer of industry includes manufacturing and construction; the third layer of industry includes financial business, commerce, service, information, and health care. The Industrial Revolution caused a shift from the first layer of industry to the second layer of industry during the 18th and 19th centuries, while the Internet Revolution and Lehman Shock promoted the prevalence of commodity-assembly manufacturing to reduce personnel expenses and material costs, causing another rotation from the second layer of industry to the third layer of industry in the 21 st century.

There are many analogies between the three-layer structure and development process of omics medicine and those of industry. Internet technology enabled the outsourcing of diagnostics and therapeutic optimization, which are known as telemedicine. Microarray and next-generation sequencing technologies concentrated the production of highthroughput data to world-class institutes or companies to reduce personnel and consumable expenses. Because the internet and high-throughput technologies are able to promote a leap from the first layer to the third layer of omics medicine, the global scientific community appears destined to move toward translational medicine.

Clinical medicine, basic medicine and translational medicine are responsible for the establishment and maintenance of bio-banks, databases and a comprehensive knowledgebase, respectively (Figure 1B). All of these aspects are mutually dependent and indispensable for clinical sequencing and molecular medicine in the era of personalized medicine. I am convinced that balanced support for clinical medicine, basic medicine and translational medicine are mandatory for the mechanistic elucidation of human diseases and the development of diagnostics and therapeutics.

\section{ACKNOWLEDGMENTS}

Masaru Katoh is supported by the National Cancer Center Research and Development Fund.

\section{REFERENCES}

Arzumanyan, A., Sambandam, V., Clayton, M. M., Choi, S. S., Xie, G., Diehl, A. M., et al. (2012). Hedgehog signaling blockade delays hepatocarcinogenesis induced by hepatitis B virus X protein. Cancer Res. 72, 5912-5920. doi: 10.1158/00085472.CAN-12-2329

Baylin, S. B., and Jones, P. A. (2011). A decade of exploring the cancer epigenome-biological and translational implications. Nat. Rev. Cancer 11, 726-734. doi: 10.1038/nrc3130

Beenken, A., and Mohammadi, M. (2009). The FGF family: biology, pathophysiology and therapy. Nat. Rev. Drug Discov. 8, 235-253. doi: $10.1038 / \mathrm{nrd} 2792$ 
Brock, H. W., and Fisher, C. L. (2005). Maintenance of gene expression patterns. Dev. Dyn. 232, 633-655. doi: $10.1002 /$ dvdy.20298

Buettner, R., Wolf, J., and Thomas, R. K. (2013). Lessons learned from lung cancer genomics: the emerging concept of individualized diagnostics and treatment. J. Clin. Oncol. 31, 1858-1865. doi: 10.1200/JCO.2012.45.9867

Carlsson, P., and Mahlapuu, M. (2002). Forkhead transcription factors: key players in development and metabolism. Dev. Biol. 250, 1-23. doi: 10.1006/dbio.2002.0780

Chien, A. J., Conrad, W. H., and Moon, R. T. (2009). A Wnt survival guide: from flies to human disease. J. Invest. Dermatol. 129, 1614-1627. doi: 10.1038/jid.2008.445

Ciardiello, F., and Tortora, G. (2008). EGFR antagonists in cancer treatment. N. Engl. J. Med. 358, 1160-1174. doi: 10.1056/NEJMra0707704

Clevers, H. (2006). Wnt/ $\beta$-catenin signaling in development and disease. Cell 127, 469-480. doi: 10.1016/j.cell.2006.10.018

Dickson, C., Smith, R., Brookes, S., and Peters, G. (1984). Tumorigenesis by mouse mammary tumor virus: proviral activation of a cellular gene in the common integration region int-2. Cell 37, 529-536. doi: 10.1016/0092-8674(84)90383-0

Ellis, L. M., and Hicklin, D. J. (2008). VEGF-targeted therapy: mechanisms of anti-tumour activity. Nat. Rev. Cancer 8, 579-591. doi: 10.1038/nrc2403

Eswarakumar, V. P., Lax, I., and Schlessinger, J. (2005). Cellular signaling by fibroblast growth factor receptors. Cytokine Growth Factor Rev. 16, 139-149. doi: 10.1016/j.cytogfr.2005.01.001

Fisher, C. L., Berger, J., Randazzo, F., and Brock, H. W. (2003). A human homolog of Additional sex combs, ADDITIONAL SEX COMBS-LIKE 1, maps to chromosome 20q11. Gene 306, 115-126. doi: 10.1016/S0378-1119(03)00430-X

Gelsi-Boyer, V., Brecqueville, M., Devillier, R., Murati, A., Mozziconacci, M. J., and Birnbaum, D. (2012). Mutations in ASXL1 are associated with poor prognosis across the spectrum of malignant myeloid diseases. J. Hematol. Oncol. 5, 12. doi: $10.1186 / 1756-8722-5-12$

Gurney, A., Axelrod, F., Bond, C. J., Cain, J., Chartier, C., Donigan, L., et al. (2012). Wnt pathway inhibition via the targeting of Frizzled receptors results in decreased growth and tumorigenicity of human tumors. Proc. Natl. Acad. Sci. U.S.A. 109, 11717-11722. doi: 10.1073/pnas.1120068109

Hannenhalli, S., and Kaestner, K. H. (2009). The evolution of Fox genes and their role in development and disease. Nat. Rev. Genet. 10, 233-240. doi: $10.1038 / \mathrm{nrg} 2523$

Jürgens, G. (1985). A group of genes controlling the spatial expression of the bithorax complex in Drosophila. Nature 316, 153-155. doi: 10.1038/316153a0

Katoh, M. (2002a). Paradigm shift in gene-finding method: from bench-top approach to desk-top approach. Int. J. Mol. Med. 10, 677-682.

Katoh, M. (2002b). WNT and FGF gene clusters. Int. J. Oncol. 21, 1269-1273.

Katoh, M. (2013a). Functional and cancer genomics of ASXL family members. Br. J. Cancer 109, 299-306. doi: $10.1038 / b j c .2013 .281$
Katoh, M. (2013b). Therapeutics targeting angiogenesis: genetics and epigenetics, extracellular miRNAs and signaling networks. Int. J. Mol. Med. 32, 763-767. doi: 10.3892/ijmm.2013.1444

Katoh, M., Igarashi, M., Fukuda, H., Nakagama, H., and Katoh, M. (2013). Cancer genetics and genomics of human FOX family genes. Cancer Lett. 328, 198-206. doi: 10.1016/j.canlet.2012.09.017

Katoh, M., and Katoh, M. (2003). Identification and characterization of ASXL2 gene in silico. Int. J. Oncol. 23, 845-850.

Katoh, M., and Katoh, M. (2004a). Human FOX gene family. Int. J. Oncol. 25, 1495-500.

Katoh, M., and Katoh, M. (2004b). Identification and characterization of ASXL3 gene in silico. Int. J. Oncol. 24, 1617-1622.

Katoh, M., and Katoh, M. (2004c). Identification and characterization of human FOXN5 and rat Foxn5 genes in silico. Int. J. Oncol. 24, 1339-1344.

Katoh, M., and Katoh, M. (2007). WNT signaling pathway and stem cell signaling network. Clin. Cancer Res. 13, 4042-4045. doi: 10.1158/10780432.CCR-06-2316

Katoh, Y., and Katoh, M. (2009). Hedgehog target genes: mechanisms of carcinogenesis induced by aberrant hedgehog signaling activation. Curr. Mol. Med. 9, 873-886. doi: $10.2174 / 156652409789105570$

Katoh, M., and Nakagama, H. (2013). FGF receptors: cancer biology and therapeutics. Med. Res. Rev. doi: 10.1002/med.21288. [Epub ahead of print].

Koike, J., Takagi, A., Miwa, T., Hirai, M., Terada, M., and Katoh, M. (1999). Molecular cloning of Frizzled-10, a novel member of the Frizzled gene family. Biochem. Biophys. Res. Commun. 262, 39-43. doi: 10.1006/bbrc.1999.1161

Kwak, E. L., Bang, Y. J., Camidge, D. R., Shaw, A. T., Solomon, B., Maki, R. G., et al. (2010). Anaplastic lymphoma kinase inhibition in non-small-cell lung cancer. N. Engl. J. Med. 363, 1693-1703. doi: 10.1056/NEJMoa1006448

Lagerström, M. C., and Schiöth, H. B. (2008). Structural diversity of $\mathrm{G}$ protein-coupled receptors and significance for drug discovery. Nat. Rev. Drug Discov. 7, 339-357. doi: 10.1038/nrd2518

Lappano, R., and Maggiolini, M. (2011). G proteincoupled receptors: novel targets for drug discovery in cancer. Nat. Rev. Drug Discov. 10, 47-60. doi: $10.1038 / \mathrm{nrd} 3320$

Lehmann, O. J., Sowden, J. C., Carlsson, P., Jordan, T., and Bhattacharya, S. S. (2003). Fox's in development and disease. Trends Genet. 19, 339-344. doi: 10.1016/S0168-9525(03)00111-2

Li, T., Kung, H. J., Mack, P. C., and Gandara, D. R. (2013). Genotyping and genomic profiling of nonsmall-cell lung cancer: implications for current and future therapies. J. Clin. Oncol. 31, 1039-1049. doi: 10.1200/JCO.2012.45.3753

Mologni, L. (2011). Development of RET kinase inhibitors for targeted cancer therapy. Curr. Med. Chem. 18, 162-175. doi: 10.2174/092986711794088308

Nusse, R., van Ooyen, A., Cox, D., Fung, Y. K., and Varmus, H. (1984). Mode of proviral activation of a putative mammary oncogene (int-1) on mouse chromosome 15. Nature 307, 131-136. doi: $10.1038 / 307131 \mathrm{a} 0$
Ordovás, J. M., and Smith, C. E. (2010). Epigenetics and cardiovascular disease. Nat. Rev. Cardiol. 7, 510-519. doi: 10.1038/nrcardio.2010.104

Penn, J. S., Madan, A., Caldwell, R. B., Bartoli, M., Caldwell, R. W., and Hartnett, M. E. (2008). Vascular endothelial growth factor in eye disease. Prog. Retin. Eye Res. 27, 331-371. doi: 10.1016/j.preteyeres.2008.05.001

Philips, G. M., Chan, I. S., Swiderska, M., Schroder, V. T., Guy, C., Karaca, G. F., et al. (2011). Hedgehog signaling antagonist promotes regression of both liver fibrosis and hepatocellular carcinoma in a murine model of primary liver cancer. PLoS ONE 6:e23943. doi: 10.1371/journal.pone. 0023943

Sagara, N., Toda, G., Hirai, M., Terada, M., and Katoh, M. (1998). Molecular cloning, differential expression, and chromosomal localization of human Frizzled-1, Frizzled-2, and Frizzled-7. Biochem. Biophys. Res. Commun. 252, 117-122. doi: 10.1006/bbrc. 1998.9607

Santo, E. E., Ebus, M. E., Koster, J., Schulte, J. H., Lakeman, A., van Sluis, P., et al. (2012). Oncogenic activation of FOXR1 by 11q23 intrachromosomal deletion-fusions in neuroblastoma. Oncogene 31 , 1571-1581. doi: 10.1038/onc.2011.344

Sexton, P. M., and Wootten, D. (2013). Structural biology: meet the B family. Nature 499, 417-418. doi: 10.1038/nature12413

Sinclair, D. A., Milne, T. A., Hodgson, J. W., Shellard, J., Salinas, C. A., Kyba, M., et al. (1998). The Additional sex combs gene of Drosophila encodes a chromatin protein that binds to shared and unique Polycomb group sites on polytene chromosomes. Development 125, 1207-1216.

Tammela, T., Enholm, B., Alitalo, K., and Paavonen, K. (2005). The biology of vascular endothelial growth factors. Cardiovasc. Res. 65, 550-563. doi: 10.1016/j.cardiores.2004.12.002

Turchinovich, A., Weiz, L., and Burwinkel, B. (2012). Extracellular miRNAs: the mystery of their origin and function. Trends Biochem. Sci. 37, 460-465. doi: 10.1016/j.tibs.2012.08.003

Wittmann, J., and Jäck, H. M. (2010). Serum microRNAs as powerful cancer biomarkers. Biochim. Biophys. Acta. 1806, 200-207.

Received: 28 August 2013; accepted: 26 September 2013; published online: 04 October 2013.

Citation: Katoh M (2013) Great challenges in molecular medicine: toward personalized medicine. Front. Cell Dev. Biol. 1:1. doi: 10.3389/fcell.2013.00001

This article was submitted to Molecular Medicine, a section of the journal Frontiers in Cell and Developmental Biology.

Copyright (c) 2013 Katoh. This is an open-access article distributed under the terms of the Creative Commons Attribution License (CC BY). The use, distribution or reproduction in other forums is permitted, provided the original author(s) or licensor are credited and that the original publication in this journal is cited, in accordance with accepted academic practice. No use, distribution or reproduction is permitted which does not comply with these terms. 